\title{
A Rule-Based Method to Model Myocardial Fiber Orientation for Simulating Ventricular Outflow Tract Arrhythmias
}

\author{
Ruben Doste ${ }^{1}$, David Soto-Iglesias ${ }^{1}$, Gabriel Bernardino ${ }^{1}$, Rafael Sebastian ${ }^{2}$, \\ Sophie Giffard-Roisin ${ }^{3}$, Rocio Cabrera-Lozoya ${ }^{3}$, Maxime Sermesant ${ }^{3}$, Antonio \\ Berruezo $^{4}$, Damian Sanchez-Quintana ${ }^{5}$, and Oscar Camara ${ }^{1}$ \\ 1 Physense, Universitat Pompeu Fabra, Barcelona, Spain \\ ruben.doste@upf .edu \\ 2 Computational Multiscale Simulation Lab (CoMMLab), Department of Computer \\ Science, Universitat de Valencia, Valencia, Spain \\ 3 Inria, Asclepios Research Group, Sophia-Antipolis, France \\ 4 Arrhythmia Section, Cardiology Department, Thorax Institute. Hospital Clinic, \\ University of Barcelona, Barcelona, Spain \\ 5 Department of Anatomy and Cell Biology, Faculty of Medicine, University of \\ Extremadura, Badajoz, Spain
}

\begin{abstract}
Myocardial fiber orientation determines the propagation of electrical waves in the heart and the contraction of cardiac tissue. One common approach for assigning fiber orientation to cardiac anatomical models are Rule-Based Methods (RBM). However, RBM have been developed to assimilate data mostly from the Left Ventricle. In consequence, fiber information from RBM does not match with histological data in other areas of the heart, having a negative impact in cardiac simulations beyond the LV. In this work, we present a RBM where fiber orientation is separately modeled in each ventricle following observations from histology. This allows to create detailed fiber orientation in specific regions such as the right ventricle endocardium, the interventricular septum and the outflow tracts. Electrophysiological simulations including these anatomical structures were then performed, with patient-specific data of outflow tract ventricular arrhythmias (OTVA) cases. A comparison between the obtained simulations and electro-anatomical data of these patients confirm the potential for in silico identification of the site of origin in OTVAs before the intervention.
\end{abstract}

Keywords: fiber orientation, rule-based method, electrophysiological simulations, arrhythmias, outflow tracts

\section{Introduction}

Outflow tract ventricular arrhythmias (OTVAs) are a type of arrhythmia in which the site of origin (SOO) of the ectopic beat is located in one of the two outflow tracts. In order to treat this disease, clinicians need to locate and ablate its SOO by Radio Frequency Ablation (RFA). Usually, the ectopic focus is 
identified after visual inspection of the electrocardiogram (ECG) by experienced observers, which is confirmed during the intervention with Electro-Anatomical Mapping (EAM) data. Unfortunately, sometimes the SOO cannot be properly determined from the ECG since it can be located in regions where both OTs are very close. In those cases, RFA interventions can last several hours if several EAMs need to be acquired to identify the SOO.

Personalized electrophysiological simulations of the heart have recently shown promising results to support clinical decisions [1]. Nevertheless, there are some limitations in the existing models for helping to determine the SOO in OTVAs. Most simulation studies in the literature have focused on the left ventricle (LV) due to the complexity of obtaining accurate data of the right ventricle (RV), especially on fiber orientation (e.g. with Diffusion Tensor Magnetic Resonance Imaging [2]). Even though biventricular geometries are often considered in the models, they do not include the outflow tracts, but an artificial basal plane well below the valves. One of the main reasons for that practice is that Rule-Based Methods (RBM) usually employed to generate myocardial fiber orientation do not include specific information about the RV or the outflow tracts, preventing the use of cardiac simulations with OTVA data.

Since electrical wave propagation is determined by fiber orientation $[3,4]$, a proper fiber configuration is needed in order to obtain more accurate and reliable simulation results. We present here an adaptation of an existing RBM [5] that includes specific fiber orientation in cardiac regions relevant for OTVAs, following observations from histological data. According to this data, fiber orientation in the RV sub-endocardium has a longitudinal direction from the apex towards the pulmonary and tricuspid valves, as illustrated in Fig. 1. Moreover, fiber orientation in the sub-endocardium and sub-epicardium of the OT have a longitudinal and circumferential direction, respectively. In addition, the developed RBM processes both ventricles independently, which gives more flexibility to generate different fiber configurations. Septal fiber orientation can also be independently modified, allowing the study of its discontinuity, which is still under debate: whereas some studies indicate the existence of a continuous septum [6], others show fiber discontinuity and evidences of a bilayered septum $[7,8]$.

In this work, fiber configurations generated with the proposed RBM are compared with state-of-the-art RBM in a simple heart geometry without OTs for verification purposes. Several electrophysiological simulations were performed to study the effect of using different fiber configurations on the heart electrical propagation. Finally, the proposed RBM was applied to several patient-specific geometries showing OTVA in which electrophysiological simulations with different SOOs were run. The resulting in silico isochrones around the RV earliest activated point were then compared with patient-specific EAM data for validation purposes. 

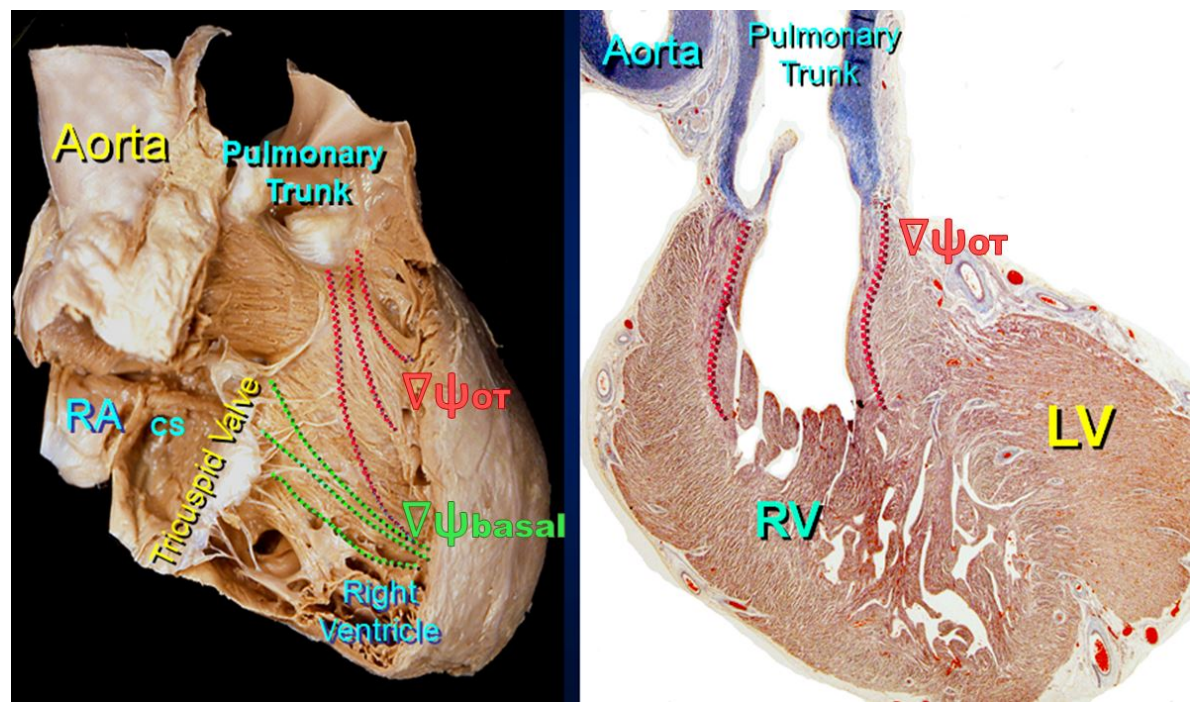

Fig. 1. Histological data of the heart. Left: Fiber configuration in the RV subendocardium, with longitudinal directions to the pulmonary and tricuspid valves (dashed red and green lines, respectively). Right: RVOT slice showing longitudinal direction in the sub-endocardium wall. R: Right; L: Left; A: atrium; V: ventricle; CS: coronary sinus; $\nabla \Psi_{\text {basal }}$ : apico-basal direction; $\nabla \Psi_{O T}$ : apico-OT direction

\section{Methodology}

The developed RBM was inspired by the work of Bayer et al. [5], which is mainly based on Streeter's observations [9] from the left ventricle. We have improved that RBM by:

i) Extending fiber orientation up to the outflow tracts.

ii) Modifying the fibers in the RV endocardium according to histological studies. These fibers have two main longitudinal directions, one from the apex to the pulmonary valve and other from the apex to the tricuspid valve (see Fig. 1).

iii) Allowing septal discontinuities by modifying the angle between the septal fibers of LV and RV.

\subsection{Mesh Generation}

The geometries used in this work were patient-specific tetrahedral meshes built from the processing of computed tomography (CT) images. A bi-ventricular model including the outflow tracts was built from semi-automatic segmentation performed by region growing techniques available in 3DSlicer ${ }^{6}$. Subsequently, surface meshes were generated applying the classical Marching Cubes method,

\footnotetext{
${ }^{6}$ https://www.slicer.org
} 
which was followed by some post-processing steps (e.g. smoothing, labelling), performed in Blender ${ }^{7}$. Finally, tetrahedral meshes ( $~ 80000$ nodes and $\sim 400000$ elements) were created using iso 2 mesh $^{8}$.

\subsection{Fiber Generation Algorithm}

A scheme of this process can be visualized in Fig. 2. The first step for assigning fiber orientation was to generate a local orthonormal reference system at each node of a given personalized heart geometry. The axes of the local orthonormal reference system were the longitudinal $\left(\hat{e}_{l}\right)$ transmural $\left(\hat{e}_{t}\right)$ and circumferential $\left(\hat{e}_{c}\right)$ directions. Transmural and longitudinal directions were defined by solving the Laplace equation using the corresponding geometrical surfaces as Dirichlet boundary conditions and computing the gradient of the resulting distribution map; the geometrical surfaces were: RV and LV endocardium; epicardium; apex; and the tricuspid, mitral, pulmonary and aortic valves. The circumferential direction was computed from the cross product of transmural and longitudinal directions. Fiber orientation was then obtained by rotating the obtained vector $\hat{e}_{c}$ around $\hat{e}_{t}$ by a given angle $\alpha$.

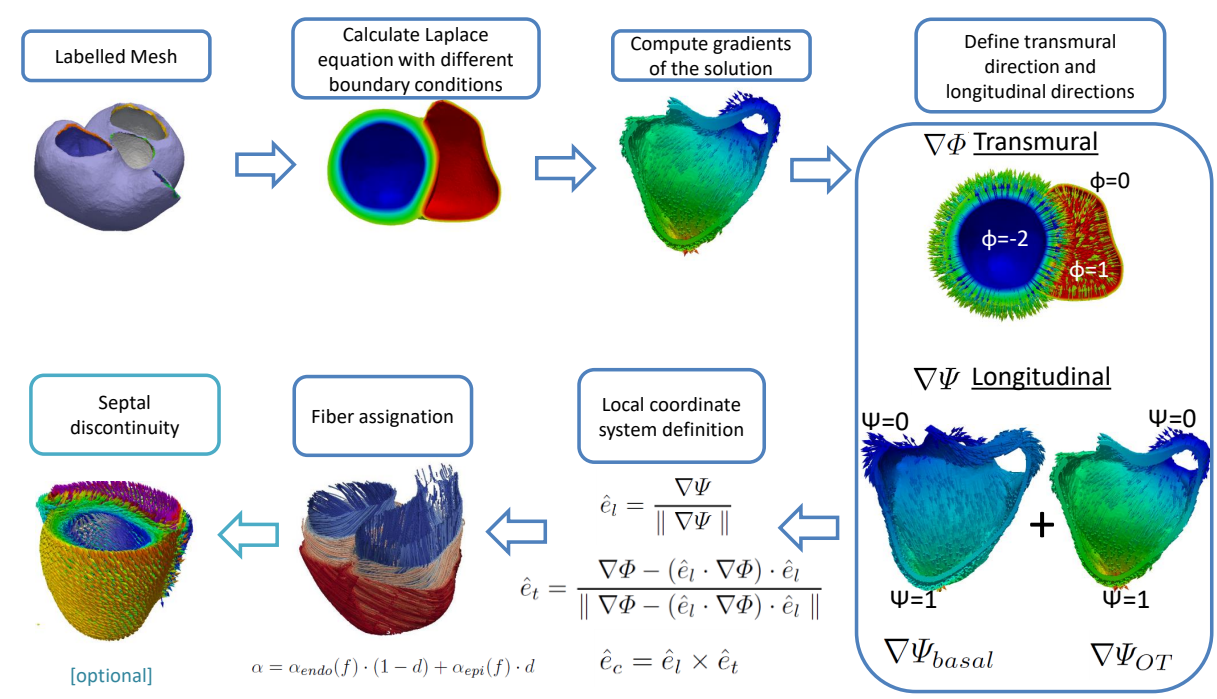

Fig. 2. Schematic showing the different steps of the presented RBM. $\nabla \Psi$ : longitudinal gradient; $\nabla \Psi_{\text {basal }}$ : apico-basal gradient; $\nabla \Psi_{O T}$ : apico-OT gradient; $\nabla \Phi$ : transmural gradient; $\hat{e}_{l}$ : longitudinal axis; $\hat{e}_{t}$ : transmural axis; $\hat{e}_{c}$ : circumferential axis.

Specifically, transmural direction $(\nabla \Phi)$ was defined solving the Laplace equation between the endocardium and epicardium of each ventricle independently,

\footnotetext{
7 https://www.blender.org

8 http://iso2mesh.sourceforge.net
} 
and subsequently computing the gradient of the Laplace solution. The Laplace equation was simultaneously computed for both ventricles, assigning a negative value to the $\mathrm{LV}$ endocardium and a positive to the RV one, allowing independent fiber configurations for both ventricles. Longitudinal direction $(\nabla \Psi)$ was also defined separately in each ventricle. This direction was the result of a weighted sum of the apico-basal gradient $\left(\nabla \Psi_{\text {basal }}\right)$ and the apico-OT gradient $\left(\nabla \Psi_{O T}\right)$, defined individually in both ventricles: $\nabla \Psi_{\text {basal }}$ considered the mitral or tricuspid valves and $\nabla \Psi_{O T}$ the aortic or pulmonary valves, for LV and RV, respectively. These two main directions were already described by Greenbaum et al. [10] and can be visualized in Fig. 1 (dashed lines). The previous sum was weighted by an intra-ventricular interpolation function $f$, which was computed by solving the Laplace equation between the pulmonary-tricuspid valve and the aortic-mitral valve in the RV and LV, respectively. In this way, we obtained a distribution of values allowing to control the smoothness in fiber changes near the OT in different geometries. The resulting longitudinal direction for each ventricle was:

$$
\nabla \Psi=\nabla \Psi_{b a s a l} \cdot f+\nabla \Psi_{O T} \cdot(1-f)
$$

Using the previously calculated gradients $(\nabla \Psi, \nabla \Phi)$, the local coordinate system was set up for each vertex, which is fully described with the following vectors:

$$
\hat{e}_{l}=\frac{\nabla \Psi}{\|\nabla \Psi\|} \quad \hat{e}_{t}=\frac{\nabla \Phi-\left(\hat{e}_{l} \cdot \nabla \Phi\right) \cdot \hat{e}_{l}}{\left\|\nabla \Phi-\left(\hat{e}_{l} \cdot \nabla \Phi\right) \cdot \hat{e}_{l}\right\|} \quad \hat{e}_{c}=\hat{e}_{l} \times \hat{e}_{t}
$$

Fiber orientation was estimated in every vertex by rotating counterclockwise the vector $\hat{e}_{c}$ around $\hat{e}_{t}$ the vector $\hat{e}_{c}$ with an angle $\alpha$, defined in each ventricle as:

$$
\alpha=\alpha_{\text {endo }}(f) \cdot(1-d)+\alpha_{\text {epi }}(f) \cdot d
$$

where $d$ is the transmural depth normalized from 0 to 1 . The different values of $\alpha_{\text {endo }}$ and $\alpha_{e p i}$ were chosen to replicate observations from several histological studies [9-11]:

- LV (based on Streeter's observations [9]): $\alpha_{\text {endo }}(f=1)=-60^{\circ} ; \alpha_{\text {epi }}(f=$ 1) $=60^{\circ}$

- RV (based on Greenbaum [10] and Sanchez-Quintana [11]): $\alpha_{\text {endo }}(f=1)=$ $90^{\circ}$ (same as longitudinal direction); $\alpha_{e p i}(f=1)=-25^{\circ}$

- OTs (based on Sanchez-Quintana [11]): $\alpha_{\text {epi }}(f=0)=0^{\circ}$ (circumferential direction) $; \alpha_{\text {endo }}(f=0)=90^{\circ}$ (longitudinal direction)

\subsection{Septal Discontinuity}

The presented RBM is able to include fiber angle discontinuity in the septum. This can be done since we define transmural direction $(\nabla \Phi)$ independently in each ventricle. The first step was to determine the contribution of each ventricle to the septal wall; some studies [7] state that two thirds of the septal wall belong 
to the LV. Therefore, we applied modified Dirichlet conditions when computing the Laplace equation: the negative values assigned to the LV were the double of the positive ones assigned to the RV surfaces. In this way, we could identify a border surface between the positive/negative values and use it for guiding the interpolation of the fiber angles in the septum. Therefore, the expression for assigning the fiber angle in the septum remained as:

$$
\alpha=\alpha_{\text {endo }}(f) \cdot(1-d)+\alpha_{\text {septal }}(f) \cdot d
$$

where $\alpha_{\text {septal }}$ is the fiber angle at the border of the ventricles. Continuity or a certain angle discontinuity in fiber orientation could easily be forced by modifying this angle.

\section{Results}

\subsection{Angle difference}

The performance of the developed method was analyzed setting up an experiment to estimate fiber angle differences with respect to Bayer's RBM [5] and several configurations of the presented RBM. However, Bayer's RBM is not able to reproduce fiber orientation above the basal plane since it is used as a boundary condition, making the comparison of the OT fibers unfeasible. Thus a bi-ventricular geometry of OTVA patients was cut below the outflow tracts so that all the existing RBM could be run. As expected, the main angle differences between both RBM were found in the RV endocardium and near the RVOT (see upper row of Fig. 3), where we forced longitudinal directions from the apex towards the pulmonary and tricuspid valves in our method.

\subsection{Electrical Activation}

The impact of the proposed RBM in the electrical activation of the heart was evaluated running several electrophysiological simulations with fiber configurations provided by the existing and proposed RBMs. Simulations were performed with the SOFA software ${ }^{9}$, using the Mitchell-Schaeffer model [12]. Using one of the available bi-ventricular geometries (shown in Fig. 3), simulations were run placing the ectopic focus in the RV apex. In each simulation only fiber orientation was changed, keeping unaltered the remaining parameters of the model such as the conductivity anisotropy ratio (in our simulations conduction velocity in the fiber direction is 2.5 larger than in the transverse plane).Lower row of the Fig. 3 shows the electrical activation isochrones obtained with the continuous and discontinuous septum versions of the proposed RBM as well as with Bayer's RBM (left, center and right in the lower row of the figure, respectively). It can be observed in Fig. 3 that the electrical activation patterns are quite similar except

\footnotetext{
${ }^{9}$ An Open Source medical simulation software http://www . sofa-framework.org
} 


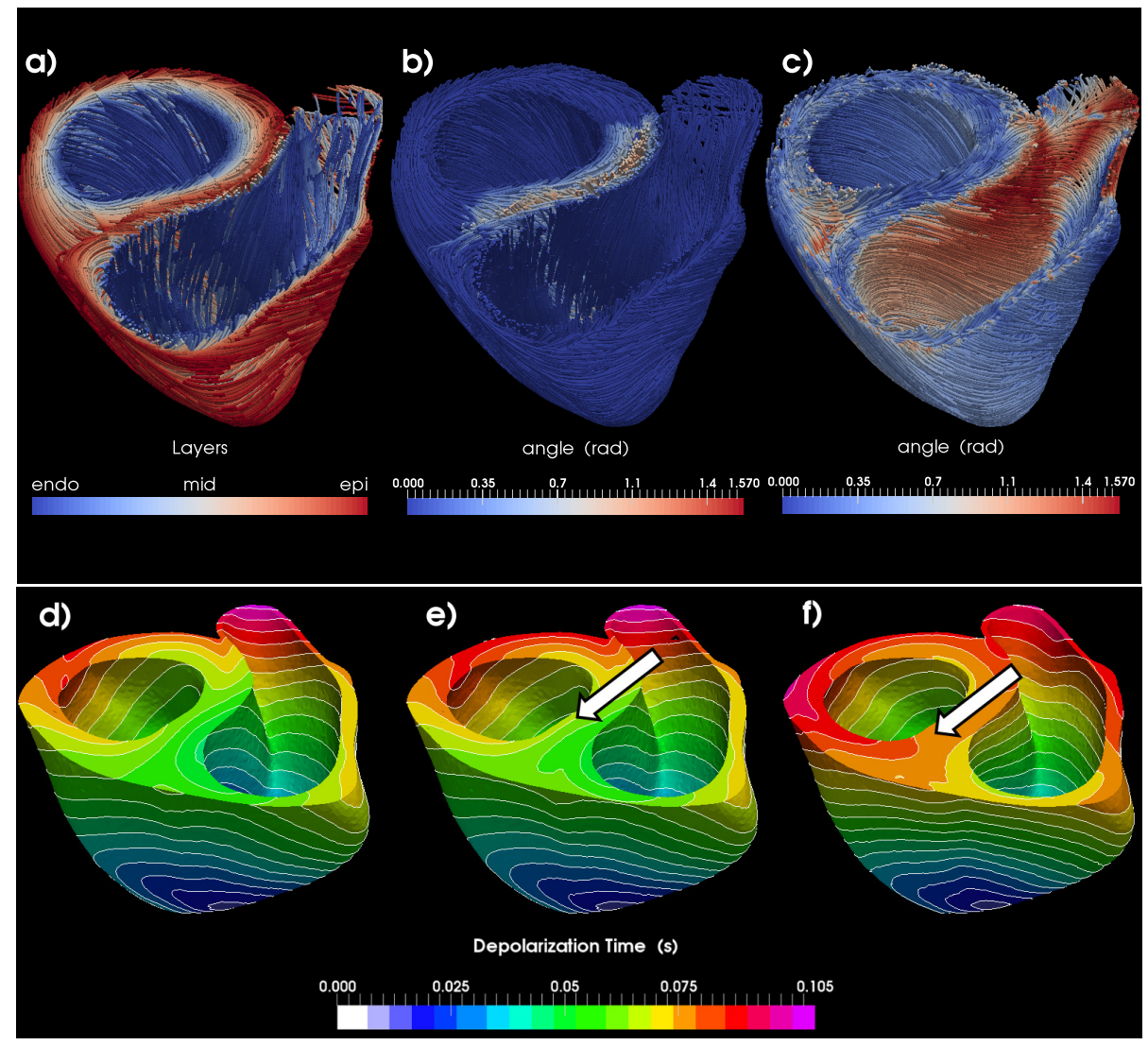

Fig. 3. Upper row:(a) Fiber orientation provided by the proposed RBM with a continuous septum; (b) angle differences (in radians) with fibers in a discontinuous septum; (c) and with Bayer's RBM method. Lower row: Electrical activation isochrones (in seconds) provided by different models: the proposed RBM with a continuous (d) and discontinuous (e) septum; f) Bayer's RBM. White arrows point towards the main differences found in the septal activation pattern. 
in the septum and the RV endocardium (see white arrows), using the continuous septum version of the proposed RBM as reference. The larger delay in the basal septum showed in panel C comparing to the ones from our method (panels A and $\mathrm{B}$ ) is due to the RV septal fiber difference. In our code, an apex-base direction was forced $\left(\nabla \Psi_{\text {basal }}\right)$, as a consequence, the impulse travels faster in our geometry than in the obtained by Bayer's RBM, which has more circumferential fibers.

These results can be evaluated in a more quantitative way by calculating the mean local activation time (LAT) differences for each geometry. Taking the continuous septum version of the proposed RBM as reference, LAT differences were: $2 \pm 2 \mathrm{~ms}$ (mean \pm std) with the discontinuous septum version; and $6 \pm 5$ ms compared with Bayer's RBM. A more localized analysis in the septal wall showed larger LAT differences: $4 \pm 2 \mathrm{~ms}$ with the discontinuous septum version; and $8 \pm 6$ ms with Bayer's RBM.

\subsection{OTVA simulations}

Lastly, we have also run electrophysiological simulations with fiber orientation computed with the proposed RBM on heart geometries of different OTVA patients. The obtained simulated electrical propagation waves were then compared with patient-specific EAM data. In particular, we analyzed the characteristics of the isochrones around the earliest activated point in the RV endocardium since they provide useful information about the SOO [13,14]: if the long axis of the isochrones is more longitudinal (i.e. follows the apico-basal axis) the SOO should be in the RVOT (following the fibers in the OT); while LVOT origins create more isotropic isochrones or with a larger perpendicular axis $[13,14]$.

Simulations were performed in heart geometries from three OTVA patients, each one with a different SOO according to clinical diagnosis: RVOT; Right Coronary Cusp (RCC) and Left Coronary Cusp (LCC) in the LVOT. The area and the axis ratio (longitudinal axis/perpendicular axis) of the simulated isochrones were compared in each case with patient data, as shown in Fig. 4 and Table 1. In agreement with clinical observations, parameters obtained from simulations confirmed that when the ectopic focus was originated in the RVOT the longitudinal diameter of the isochrones was larger than the cross-fiber one. By contrast, LVOT origins were associated with a larger perpendicular diameter, making the diameter ratio smaller. Table 1 shows how the obtained ratios of the RVOT isochrones are bigger than 1 (longer longitudinal axis) whereas the LVOT isochrones had a ratio smaller than 1 . Nevertheless, we found substantial differences in isochronal areas, that could be caused by the difficulties for measuring them and the effect of far field in EAM data.

\section{Discussion and Conclusions}

We have presented a RBM that includes fiber information specific to the RV, interventricular septum and both OTs of the ventricles, to replicate histological 


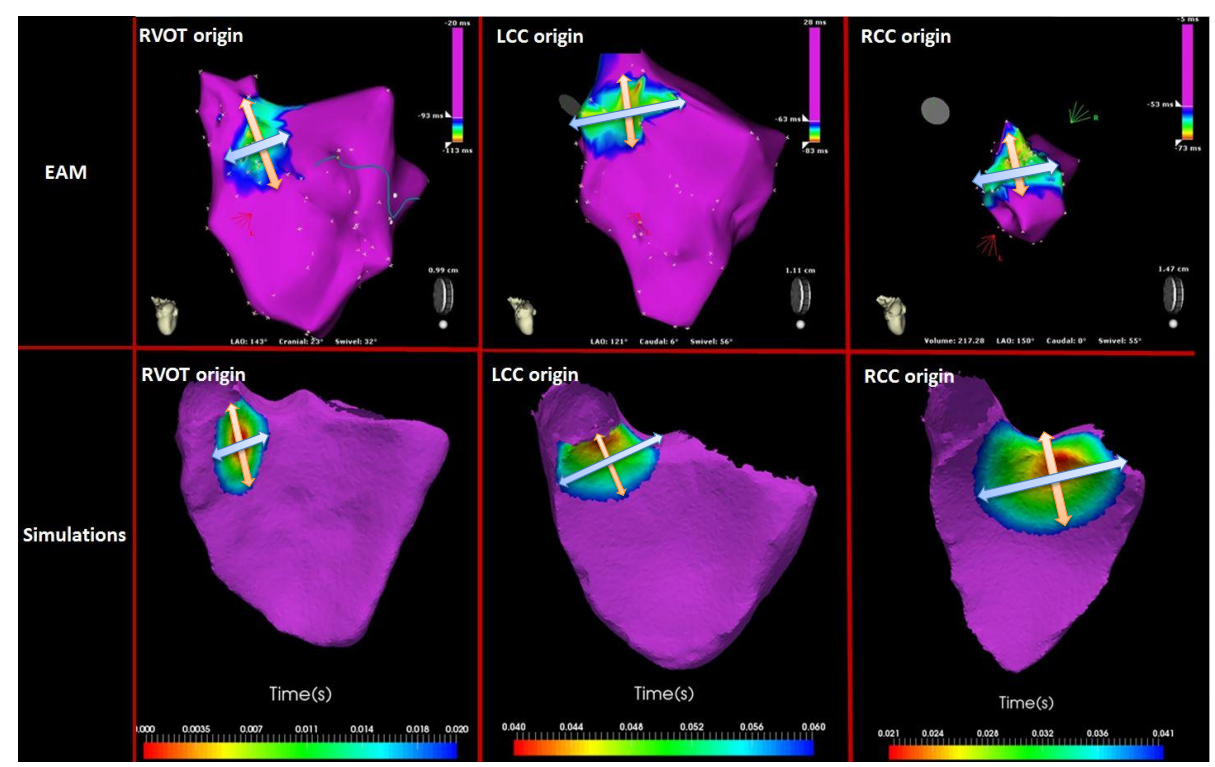

Fig. 4. Isochronal maps obtained from EAMs after $20 \mathrm{~ms}$ from earliest activated point in the RV (top) and corresponding electrophysiological simulations (bottom) in cases with different SOO. Orange and blue arrows indicate the longitudinal and perpendicular axis of the isochrones respectively. RVOT: right ventricular outflow tract. LCC and RCC: left and right coronary cusp, respectively.

Table 1. Area $\left(\mathrm{mm}^{2}\right)$ and longitudinal/perpendicular axis ratio of the $10 \mathrm{~ms}$ and 20 ms isochrones obtained from simulations and EAM data from three cases.

\begin{tabular}{c|cc|cc|cc} 
Isochrones & \multicolumn{2}{|c|}{ RVOT origin } & \multicolumn{2}{c|}{ LCC origin } & \multicolumn{2}{c}{ RCC origin } \\
& $10 \mathrm{~ms}$ & $20 \mathrm{~ms}$ & $10 \mathrm{~ms}$ & $20 \mathrm{~ms}$ & $10 \mathrm{~ms}$ & $20 \mathrm{~ms}$ \\
\hline Area Exp & $490 \pm 80$ & $990 \pm 140$ & $260 \pm 90$ & $1340 \pm 150$ & $500 \pm 100$ & $2600 \pm 250$ \\
Area Sim & $160 \pm 30$ & $540 \pm 40$ & $270 \pm 30$ & $880 \pm 50$ & $650 \pm 40$ & $2110 \pm 70$ \\
\hline Long/Perp ratio Exp & $2.04 \pm 0.7$ & $1.5 \pm 0.3$ & $0.5 \pm 0.14$ & $0.87 \pm 0.13$ & $0.56 \pm 0.14$ & $0.92 \pm 0.1$ \\
Long/Perp ratio Sim & $1.8 \pm 0.2$ & $1.6 \pm 0.1$ & $0.45 \pm 0.04$ & $0.6 \pm 0.03$ & $0.71 \pm 0.04$ & $0.72 \pm 0.03$
\end{tabular}

observations. The proposed model allows running electrophysiological simulations in applications when these regions are important such as in OTVA patients, which was not possible before. The method has been indirectly validated comparing the results of OTVA simulations (using fiber orientation provided by the proposed RBM) with patient EAM data, showing good agreement. Nevertheless, further studies are required to better determine the accuracy of the proposed RBM, such as a more quantitative comparison between RBM-derived fibers and the ones observed in histology. Additionally, OTVA simulations with RBM-derived fibers and their validation with EAM data need to be performed on a larger database of cases. It would also be desirable to compare RBM results with in-vivo cardiac diffusion imaging, which starts to provide very promising 
results $[15,16]$. This would eventually allow to overcome the existing RBM limitations to reproduce patient-specific alterations of the myofiber distribution due to infarcts or different pathologies. Future work will be devoted to analyze the impact of the new fiber configurations in mechanical simulations of the heart.

\section{Acknowledgments}

This work was partially funded by the European Union under the Horizon 2020 Programme for Research, Innovation (grant agreement No. 642676 CardioFunXion)

\section{References}

1. H. J. Arevalo, et al.: "Arrhythmia risk stratification of patients after myocardial infarction using personalized heart models," Nat Commun, vol. 7, no. May, p. 11437, 2016.

2. H. Lombaert, et al.: "Human atlas of the cardiac fiber architecture: Study on a healthy population," IEEE Trans. Med. Imaging, vol. 31, no. 7, pp. 1436--1447, 2012.

3. R. J. Young and A.V. Panfilov, "Anisotropy of wave propagation in the heart can be modeled by a Riemannian electrophysiological metric.," Proc. Natl. Acad. Sci. U. S. A., vol. 107, no. 34, pp. 15063-15068, 2010.

4. D. A. Hooks, et al., "Laminar arrangement of ventricular myocytes influences electrical behavior of the heart," Circ. Res., vol. 101, no. 10, pp. 103-113, 2007.

5. J. D. Bayer, et al.: "A novel rule-based algorithm for assigning myocardial fiber orientation to computational heart models". Ann Biomed Eng, vol. 40, no. 10, pp. $2243-2254,2012$.

6. P.Agger, et al.. "Insights from echocardiography, magnetic resonance imaging, and microcomputed tomography relative to the mid-myocardial left ventricular echogenic zone". Echocardiography, vol. 33, no. 10, pp. 1546-1556, 2016.

7. P. Boettler, et al.: "New aspects of the ventricular septum and its function: an echocardiographic study." Heart, vol. 91, no. 10, pp. 1343--1348, 2005.

8. M. J. Kocica, et al: "The helical ventricular myocardial band: global, threedimensional, functional architecture of the ventricular myocardium." Eur. J. Cardiothoracic Surg. vol. 29, no. SUPPL. 1, 2006.

9. D. D. Streeter,et al.: "Fiber orientation in the canine left ventricle during diastole and systole." Circ. Res., vol. 24, no. 3, pp. 339-347, 1969.

10. R. A. Greenbaum, et al. : "Left ventricular fibre architecture in man." Br. Heart J., vol. 45, no. 1980, pp. 248-263, 1981.

11. D. Sanchez-Quintana, et al:: "Anatomical Basis for the Cardiac Interventional Electrophysiologist". BioMed Res Int Ao, vol. 2015, 2015 .

12. H. Talbot, et al.. "Towards an interactive electromechanical model of the heart." Interface Focus, vol. 3, no. 2, 2013.

13. J. Acosta, et al.: "Impact of earliest activation site location in the septal right ventricular outflow tract for identification of left vs right outflow tract origin of idiopathic ventricular arrhythmias." Heart. Rhythm, vol. 12, no. 4, pp. 726-734, 2015 . 
14. C. Herczku, et al.: "Mapping data predictors of a left ventricular outflow tract origin of idiopathic ventricular tachycardia with V3 transition and septal earliest activation." Circ. Arrhythm. Electrophysiol., vol. 5, no. 3, pp. 484-91, Jun. 2012.

15. N. Toussaint, et al., "In vivo human cardiac fibre architecture estimation using shape-based diffusion tensor processing," Med. Image Anal., vol. 17, no. 8, pp. 1243$-1255,2013$.

16. C. Mekkaoui, et al., "Diffusion Tractography of the Entire Left Ventricle by Using Free-breathing Accelerated Simultaneous Multisection Imaging," Radiology, vol. 0, no. 0, p. 152613, 2016. 